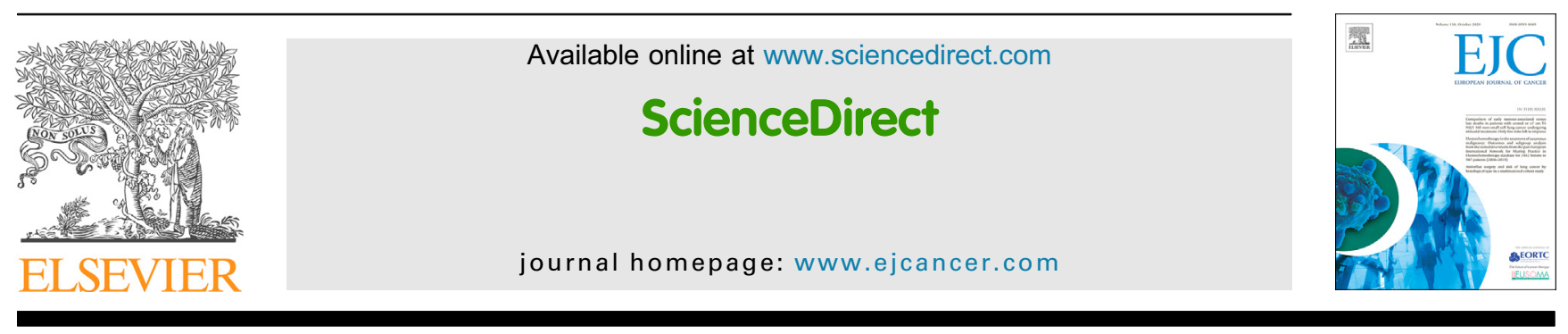

Original Research

\title{
Child development at 6 years after maternal cancer diagnosis and treatment during pregnancy
}

Tineke Vandenbroucke ${ }^{\mathrm{a}, \mathrm{b}, 1}$, Magali Verheecke ${ }^{\mathrm{a}, \mathrm{b}, 1}$, Mathilde van Gerwen ${ }^{\text {c,d,1 }}$, Kristel Van Calsteren ${ }^{\text {a,e }}$, Michael J. Halaska ${ }^{\text {f,g }}$, Monica Fumagalli h,i, Robert Fruscio ${ }^{\mathrm{j}}$, Amarendra Gandhi ${ }^{\mathrm{k}, 1}$, Margreet Veening ${ }^{\mathrm{d}}$, Lieven Lagae ${ }^{\mathrm{m}}$, Petronella B. Ottevanger ${ }^{\mathrm{n}}$, Jens-Uwe Voigt ${ }^{\circ}$, Jorine de Haan ${ }^{p}$, Mina M. Gziri ${ }^{\mathrm{q}}$, Charlotte Maggen ${ }^{\mathrm{a}, \mathrm{b}}$, Luc Mertens ${ }^{\mathrm{r}}$, Gunnar Naulaers s, Laurence Claes ${ }^{\mathrm{t}}$, Frédéric Amant ${ }^{\mathrm{b}, \mathrm{c}, \mathrm{u}, *}$ on behalf of The International Network on Cancer, Infertility and Pregnancy (INCIP) ${ }^{2}$

a Department of Obstetrics and Gynecology, University Hospitals Leuven, Leuven, Belgium

b Department of Oncology, KU Leuven, Leuven, Belgium

c Center for Gynecologic Oncology, Netherlands Cancer Institutel Antoni van Leeuwenhoek Hospital, Amsterdam, the Netherlands

d Princess Máxima Center for Pediatric Oncology, Utrecht, the Netherlands

e Department of Development and Regeneration, KU Leuven, Leuven, Belgium

${ }^{\mathrm{f}}$ Department of Obstetrics and Gynecology, 3rd Medical Faculty Charles University, Prague, Czech Republic

${ }^{g}$ Faculty Hospital Kralovske Vinohrady, Prague, Czech Republic

h Neonatal Intensive Care Unit, Fondazione IRCCS Ca' Granda Ospedale Maggiore Policlinico, Milan, Italy

${ }^{\mathrm{i}}$ Department of Clinical Sciences and Community Health, University of Milan, Milan, Italy

${ }^{\mathrm{j}}$ Clinic of Obstetrics and Gynecology, University of Milan-Bicocca and San Gerardo Hospital, Monza, Italy

${ }^{\mathrm{k}}$ Department of Public Health and Primary Care, Faculty of Medicine, KU Leuven, Leuven, Belgium

${ }^{1}$ Data Scientist, Knowledge Center, SD Worx, Antwerp, Belgium

${ }^{\mathrm{m}}$ Department of Pediatrics, University Hospitals Leuven, Leuven, Belgium

${ }^{\mathrm{n}}$ Department of Internal Medicine, Radboud UMC Nijmegen, the Netherlands

o Department of Cardiology, University Hospitals Leuven, Leuven, Belgium

p Department of Obstetrics and Gynecology, Amsterdam UMC, Location VU University Medical Center, the Netherlands

q Department of Obstetrics, Cliniques Universitaires St Luc, Brussels, Belgium

${ }^{\mathrm{r}}$ Department of Cardiology, The Hospital for Sick Children, University of Toronto, Toronto, ON, Canada

s Department of Neonatology, University Hospitals Leuven, Leuven, Belgium

${ }^{\mathrm{t}}$ Faculty of Psychology and Educational Sciences, KU Leuven, Leuven, Belgium

u Department of Obstetrics and Gynecology, Amsterdam UMC, Location Amsterdam Medical Center and University of Amsterdam, the Netherlands

\footnotetext{
* Corresponding author: Division of Gynecologic Oncology, University Hospitals Leuven, and Department of Oncology, KU Leuven, Belgium, Herestraat 49, 3000 Leuven, Belgium.

E-mail address: frederic.amant@uzleuven.be (F. Amant).

1 Contributed equally as co-first authors. ${ }^{2}$ The members of the collaboration group - The International Network on Cancer, Infertility and Pregnancy (INCIP) are listed in Acknowledgements section.
} 
Received 6 March 2020; received in revised form 15 June 2020; accepted 2 July 2020

Available online 25 August 2020

\section{KEYWORDS}

Child development;

Pregnancy;

High risk;

Infant;

Follow-up studies;

Antineoplastic agents;

Prenatal exposure

delayed effects

\begin{abstract}
Background: Data on the long-term effects of prenatal exposure to maternal cancer and its treatment on child development are scarce.

Methods: In a multicenter cohort study, the neurologic and cardiac outcomes of 6-year-old children born to women diagnosed with cancer during pregnancy were compared with the outcome of children born after an uncomplicated pregnancy. Assessment included clinical evaluation, comprehensive neuropsychological testing, electrocardiography and echocardiography.
\end{abstract}

Results: In total, 132 study children and 132 controls were included. In the study group, 97 children $(73.5 \%)$ were prenatally exposed to chemotherapy (alone or in combination with other treatments), $14(10.6 \%)$ to radiotherapy (alone or in combination), $1(0.8 \%)$ to trastuzumab, $12(9.1 \%)$ to surgery alone and $16(12.1 \%)$ to no treatment. Although within normal ranges, statistically significant differences were found in mean verbal IQ and visuospatial long-term memory, with lower scores in the study versus control group (98.1, 95\% confidence interval [CI]: 94.5-101.8, versus 104.4, 95\% CI: 100.4-108.4, P $=0.001, \mathrm{Q}<0.001$ [Q refers to the false discovery rate adjusted $\mathrm{P}$ value], and $3.9,95 \% \mathrm{CI}: 3.6-4.3$, versus $4.5,95 \% \mathrm{CI}: 4.1$ $-4.9, \mathrm{P}=0.005, \mathrm{Q}=0.045$, respectively). A significant difference in diastolic blood pressure was found, with higher values in chemotherapy-exposed $(61.1,95 \% \mathrm{CI}: 59.0$ to 63.2$)$ versus control children $(56.0,95 \%$ CI 54.1 to 57.8$)(\mathrm{P}<0.001, \mathrm{Q}<0.001)$ and in a subgroup of 59 anthracycline-exposed $(61.8,95 \%$ CI: 59.3 to 64.4$)$ versus control children $(55.9,95 \% \mathrm{CI}$ : 53.6 to 58.1$)$ ( $\mathrm{P}<0.001, \mathrm{Q}=0.02)$.

Conclusions: Children prenatally exposed to maternal cancer and its treatment are at risk for lower verbal IQ and visuospatial long-term memory scores and for higher diastolic blood pressure, but other cognitive functions and cardiac outcomes were normal at the age of 6 years. Clinical trial registration: The study is registered at ClinicalTrials.gov, NCT00330447.

(C) 2020 The Author(s). Published by Elsevier Ltd. This is an open access article under the CC BY-NC-ND license (http://creativecommons.org/licenses/by-nc-nd/4.0/).

\section{Introduction}

Cancer during pregnancy is a challenge, as the health of both the mother and foetus has to be considered in therapeutic decision-making. Over the past 20 years, clinical management of pregnant cancer patients has evolved with a higher number of patients receiving treatment during pregnancy, less terminations of pregnancy and less medically induced preterm deliveries [1]. Cancer treatment may have acute and/or chronic sideeffects on the foetus, including neurotoxicity and cardiotoxicity, as chemotherapy may cross the placenta in varying amounts [2,3]. In addition, cancer may be accompanied by maternal stress, inflammatory reactions, exposure to radiation, anaesthetic agents and other medications, potentially influencing foetal development. Notwithstanding, data on the short- and longterm impact on foetal development are still limited [4].

Our group previously published two studies, documenting reassuring health status, cognitive and cardiac outcomes at a median age of 22 months [5,6]. However, cognitive problems may become more apparent at school age and can be more accurately evaluated at older ages. Moreover, cardiac problems may develop many years after chemotherapy exposure [7,8]. Therefore, this study aims to investigate the health status and cognitive and cardiac outcome of 6-year-old children prenatally exposed to maternal cancer and its treatment and, in particular, to chemotherapy.

\section{Material and methods}

\subsection{Study participants}

This is a multicenter cohort study including children born to women diagnosed with cancer during pregnancy (with or without treatment during pregnancy) (study group). At predefined ages (1.5, 3, 6, 9, 12, 15 and 18 years), the children are invited for follow-up. In this study, we compare the outcome of 6-year-old children from the study group with children born after an uncomplicated pregnancy (control group). Study children were identified and enrolled prospectively (during pregnancy or between birth and 6 years) and evaluated 
at six referral centres in Belgium, the Netherlands, the Czech Republic and Italy, all members of the International Network on Cancer, Infertility and Pregnancy. Control children were identified and enrolled at the age of 6 years. For the neurocognitive tests and health examination, the study and control children were 1:1 matched for country, gender, age, gestational age at birth and language of the tests. For the cardiac examinations, children were 1:1 matched for gender and age. The study design and recruitment are summarised in Fig. 1. Details on the recruitment and exclusion criteria are provided in the Methods section in the data article. Ethical approval was obtained by each institution, and the parents of each child provided written informed consent to participate. The full study protocol is available at http://www.cancerinpregnancy.org/studyprotocols.

\subsection{Study testing and outcomes}

Oncological, obstetrical and neonatal data were collected. Cognitive development was examined using a comprehensive neuropsychological test battery to assess intelligence, memory, attention and behaviour problems (Table 1).
Cardiac evaluation included a 12-lead electrocardiogram and an echocardiographic examination performed according to American Society of Echocardiography guidelines. The primary cardiac outcome was left ventricular shortening fraction measured by M-mode. Secondary outcomes included the cardiac chamber dimensions (left and right ventricular end diastolic diameter, left ventricular posterior wall thickness, interventricular septum thickness), left ventricular ejection fraction, the mitral valve $\mathrm{E}$ and $\mathrm{A}$ velocity and $\mathrm{E} / \mathrm{A}$ ratio, tissue Doppler imaging velocities at the left and right ventricular wall and interventricular septum and the longitudinal and circumferential 2D-strain measurements by speckle-tracking echocardiography.

Study children underwent a clinical neurological and general paediatric examination, and the parents filled out a health questionnaire. The incidence rates of health problems were considered as secondary outcomes.

Details on the neuropsychological and echocardiographic protocol and the health questionnaire are provided in the Methods section in the data article.

\subsection{Statistical analysis}

We converted raw scores into standardised scores for the intelligence tests and behaviour questionnaires,

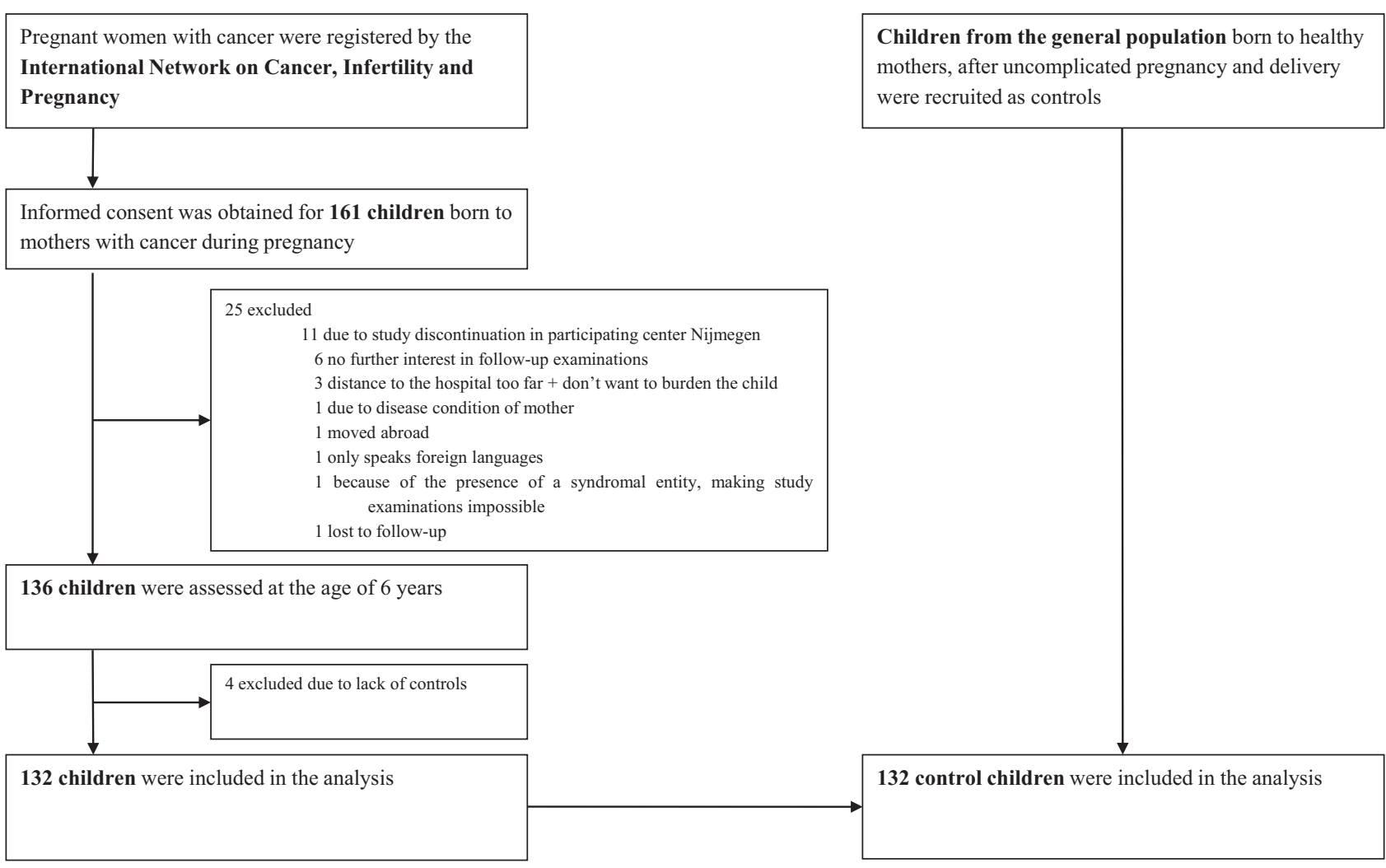

Fig. 1. Study design and recruitment. This cohort of 132 children evaluated at the age of 6 years includes 83 children who underwent cognitive evaluation and 28 children who underwent cardiac examinations in our previously published 1.5-3 years cohort study [6]. The results of 12 children at the age of 6 years were previously published [5], whereas 120 children underwent new testing. Longitudinal analyses will be performed when we reach our sample size in the oldest age group. 
Table 1

Neuropsychological outcome measures.

\begin{tabular}{|c|c|}
\hline Outcome measure & Test used \\
\hline \multicolumn{2}{|l|}{ Primary outcome } \\
\hline Full scale intelligence & $\begin{array}{l}\text { Wechsler Preschool and Primary Scale of } \\
\text { Intelligence-third edition (WPPSI-III) [26] }\end{array}$ \\
\hline \multicolumn{2}{|l|}{ Secondary outcomes } \\
\hline Verbal intelligence, performance intelligence and processing speed & $\begin{array}{l}\text { Wechsler Preschool and Primary Scale of } \\
\text { Intelligence-third edition (WPPSI-III) [26] }\end{array}$ \\
\hline $\begin{array}{l}\text { Verbal and visuospatial memory span, visuospatial short- and } \\
\text { long-term memory, verbal working memory and short- and } \\
\text { long-term memory for faces }\end{array}$ & Children's Memory Scale (CMS) [27] \\
\hline Alertness, divided attention, selective attention and response inhibition & Amsterdam Neuropsychological Tasks (ANT) [28] \\
\hline Internalising and externalising behaviour problems & Child Behavior Checklist (CBCL) [29] \\
\hline
\end{tabular}

Table 2

Cancer treatment during pregnancy for all children and those categorised as small for gestational age in singleton pregnancies.

\begin{tabular}{lll}
\hline Cancer treatment & $\begin{array}{l}\text { All children }(\mathrm{N}=132) \\
\text { Number }(\%)\end{array}$ & $\begin{array}{l}\text { Small for gestational age }(\mathrm{N}=18) \\
\text { Number (\% of children with treatment, excluding twins) }\end{array}$ \\
\hline Surgery & $12(9.1)^{\mathrm{a}}$ & $1(10.0)$ \\
Chemotherapy & $38(28.8)$ & $9(23.7)$ \\
Radiotherapy & $1(0.8)$ & $0(0.0)$ \\
Surgery and chemotherapy & $51(38.6)^{\mathrm{a}}$ & $5(11.1)$ \\
Surgery and radiotherapy & $5(3.8)$ & $2(40.0)$ \\
Surgery, chemotherapy and radiotherapy & $8(6.1)^{\mathrm{a}}$ & $0(0.0)$ \\
Trastuzumab & $1(0.8)$ & $0(0.0)$ \\
No treatment & $16(12.1)$ & $1(6.3)$
\end{tabular}

${ }^{a}$ One pair of twins was exposed to surgery alone, three pairs of twins to surgery and chemotherapy and one pair of twins to surgery, chemotherapy and radiotherapy.

based on normative data for each country provided by the test. For the memory tests, raw subtest-scores were used. Reaction times and percentage of errors were obtained for the attention tasks. Univariate analyses of covariance (ANCOVA) were used to investigate between-group differences in cognitive outcome and behaviour with education levels of parents as covariates. A subgroup analysis was performed in chemotherapy-exposed children versus controls. In addition, the incidence of behaviour problems was compared with matched controls for children whose mothers died and for those with surviving mothers. Post hoc, verbal IQ was compared between children exposed to different types of chemotherapy and their matched controls and between children whose mothers died versus those with surviving mothers and their matched controls. The associations between cognitive outcome and gestational age or the number of chemotherapy cycles were investigated using Pearson correlations. The Spearman's rank-correlation coefficient was used to investigate the relationship between cognitive outcome and the estimated foetal dose of radiation.

Echocardiographic measures were obtained in three cardiac cycles and averaged. Between-group differences were investigated using univariate analysis of variance
(ANOVA). A subgroup analysis was performed in anthracycline-exposed children versus controls.

$Q$ values, which represent false discovery rate adjusted $P$ values, were calculated to correct for multiple testing [9]. A two-sided $Q$ value of less than 0.05 was considered to indicate statistical significance.

\section{Results}

\subsection{Characteristics of the children}

In total, 132 children (including five pairs of dizygotic twins) born to mothers diagnosed with cancer during pregnancy were included, of whom 88 from Belgium, 25 from the Netherlands, 12 from Italy and 7 from the Czech Republic. During pregnancy, 97 children (73.5\%) were exposed to chemotherapy (alone or in combination with other treatments), $14(10.6 \%)$ to radiotherapy (alone or in combination), $1(0.8 \%)$ to trastuzumab, 12 $(9.1 \%)$ to surgery alone and 16 children $(12.1 \%)$ were born to mothers not treated during pregnancy (Table 2). Twenty-five mothers (19.7\%) died before the child was 6 years old. Additional information about the maternal cancer types and specific treatments is provided in Tables 1, 2 and eTable 1-6, all in the data article. 
Table 3

Demographic characteristics of the children included in cognitive and health examinations.

\begin{tabular}{|c|c|c|c|}
\hline Characteristic & Cancer during pregnancy group $(\mathrm{N}=132)$ & Control group $(\mathrm{N}=132)$ & $\mathrm{P}$ value \\
\hline Median age (range) - years & $6.1(4.8-7.9)$ & $6.2(4.7-7.7)$ & 0.29 \\
\hline Median gestational age (range) - weeks & $36.1(27.4-40.7)$ & $36.1(28.6-41.0)$ & 0.65 \\
\hline Median birth weight (range) - grams & $2705(720-4200)$ & $2713(1025-4400)$ & 0.73 \\
\hline Median maternal age at birth of this child (range) - years & $33(19-44)$ & $31(20-46)$ & 0.02 \\
\hline Sex - number $(\%)$ & & & 1.00 \\
\hline Male & $71(53.8 \%)$ & $71(53.8 \%)$ & \\
\hline Female & $61(46.2 \%)$ & $61(46.2 \%)$ & \\
\hline White & $115(87.1 \%)$ & $119(90.2 \%)$ & \\
\hline Black & $11(8.3 \%)$ & $5(3.8 \%)$ & \\
\hline Other & $6(4.5 \%)$ & $8(6.1 \%)$ & \\
\hline \multicolumn{4}{|l|}{ Highest level of education of parents - number $(\%)^{b}$} \\
\hline Mother & & & 0.07 \\
\hline Primary school & $5(3.8 \%)$ & $2(1.5 \%)$ & \\
\hline Secondary school & $52(39.4 \%)$ & $34(25.8 \%)$ & \\
\hline Master's degree or higher & $33(25.0 \%)$ & $41(31.1 \%)$ & \\
\hline Unknown & $0(0.0 \%)$ & $2(1.5 \%)$ & \\
\hline Father & & & 0.69 \\
\hline Primary school & $7(5.3 \%)$ & $5(3.8 \%)$ & \\
\hline Secondary school & $58(43.9 \%)$ & $51(38.6 \%)$ & \\
\hline Bachelor's degree & $33(25.0 \%)$ & $32(24.2 \%)$ & \\
\hline Master's degree or higher & $32(24.2 \%)$ & $39(29.5 \%)$ & \\
\hline Unknown & $2(1.5 \%)$ & $5(3.8 \%)$ & \\
\hline
\end{tabular}

a Race was self-reported by the parents.

b The highest level of education is presented according to the European educational system. A bachelor's degree is earned at both traditional universities and non-university institutions of higher education and requires between three and four years of full-time study. A master's degree is earned at university and requires one to two years of full-time study after a bachelor's degree.

In general, demographic and perinatal characteristics were comparable between the study and control group (Table 3 and eTable 7-10 in the data article).

\subsection{Perinatal outcome and growth}

In the cancer group, median gestational age at birth was 36.1 weeks (range: 27.4-40.7) and median birth weight was $2705 \mathrm{~g}$ (range: $720-4200)$. Eighty children $(60.6 \%)$ were born preterm (versus $6.8-8.0 \%$ in the participating countries) [10], of whom $8(6.1 \%)$ very preterm (27.0-31.9 weeks gestational age), $16(12.1 \%)$ moderately preterm (32.0-33.9 weeks) and $56(42.4 \%)$ late preterm $(34.0-36.9$ weeks) and 52 children $(39.4 \%)$ were born at term (37.0 weeks or later). The number and type of the registered congenital malformations were not different from the general population (eTable 11 in the data article). After exclusion of twins, $18 / 121$ children (14.9\%) in the study group were born small for gestational age (i.e. a birth weight below the tenth percentile of genderand gestational age-matched children) versus 7/119 $(5.9 \%)$ in the control group (eTable 12 in the data article). Biometric data at 6 years were comparable between the groups (eFigs. 1 and 2 in the data article).

\subsection{Cognitive development and behaviour}

Median age at cognitive evaluation was 6.1 and 6.2 years in the study and control group, respectively. The difference in estimated marginal means of the primary outcome full scale IQ was not statistically significant between the study group $(98.9,95 \%$ confidence interval [CI]: 95.2 to 102.6) and the control group (103.0, 95\% CI: 98.9 to 107.0) $(\mathrm{P}=0.03, \mathrm{Q}=0.15)$ (eTable 13 and eFig. 3 in the data article) or between the subgroup of chemotherapy-exposed children (101.4, $95 \%$ CI: 93.7 to 109.1) and controls (106.0, 95\% CI: 98.0 to 114.0$)$ $(\mathrm{P}=0.04, \mathrm{Q}=0.17$ ) (Fig. 2A) (eTable 14 in the data article). Full scale IQ was not related to gestational age in the chemotherapy-exposed group $(\mathrm{r}=-0.04$, $\mathrm{P}=0.74)$ and the control group $(\mathrm{r}=-0.08, \mathrm{P}=0.43)$ (Fig. 2B), to the number of chemotherapy cycles $(\mathrm{r}=0.04, \mathrm{P}=0.74)$ (Fig. $2 \mathrm{C}$ ) or to the dose of radiation $(\mathrm{r}=0.19, \mathrm{P}=0.52)$ (eFig. 4 in the data article).

With regard to the secondary outcomes, the difference in estimated marginal means of verbal IQ was statistically significant, with lower values in the study group (98.1, 95\% CI: 94.5 to 101.8) than the control group (104.4, 95\% CI: 100.4 to 108.4$)(\mathrm{P}=0.001$, $\mathrm{Q}<0.001)$ and in chemotherapy-exposed children (101.7, 95\% CI: 94.3 to 109.0$)$ than their matched controls $(108.5,95 \% \mathrm{CI}: 100.8$ to 116.1$)(\mathrm{P}=0.002$, $\mathrm{Q}=0.03)$. Post hoc, we evaluated the possible impact of death of the mother on verbal IQ. The size of the between-group difference in verbal IQ was larger in children whose mother died (15.1 IQ points) than those with surviving mothers (4.9 points) (eTable 16 in the data article). There were no statistically significant 

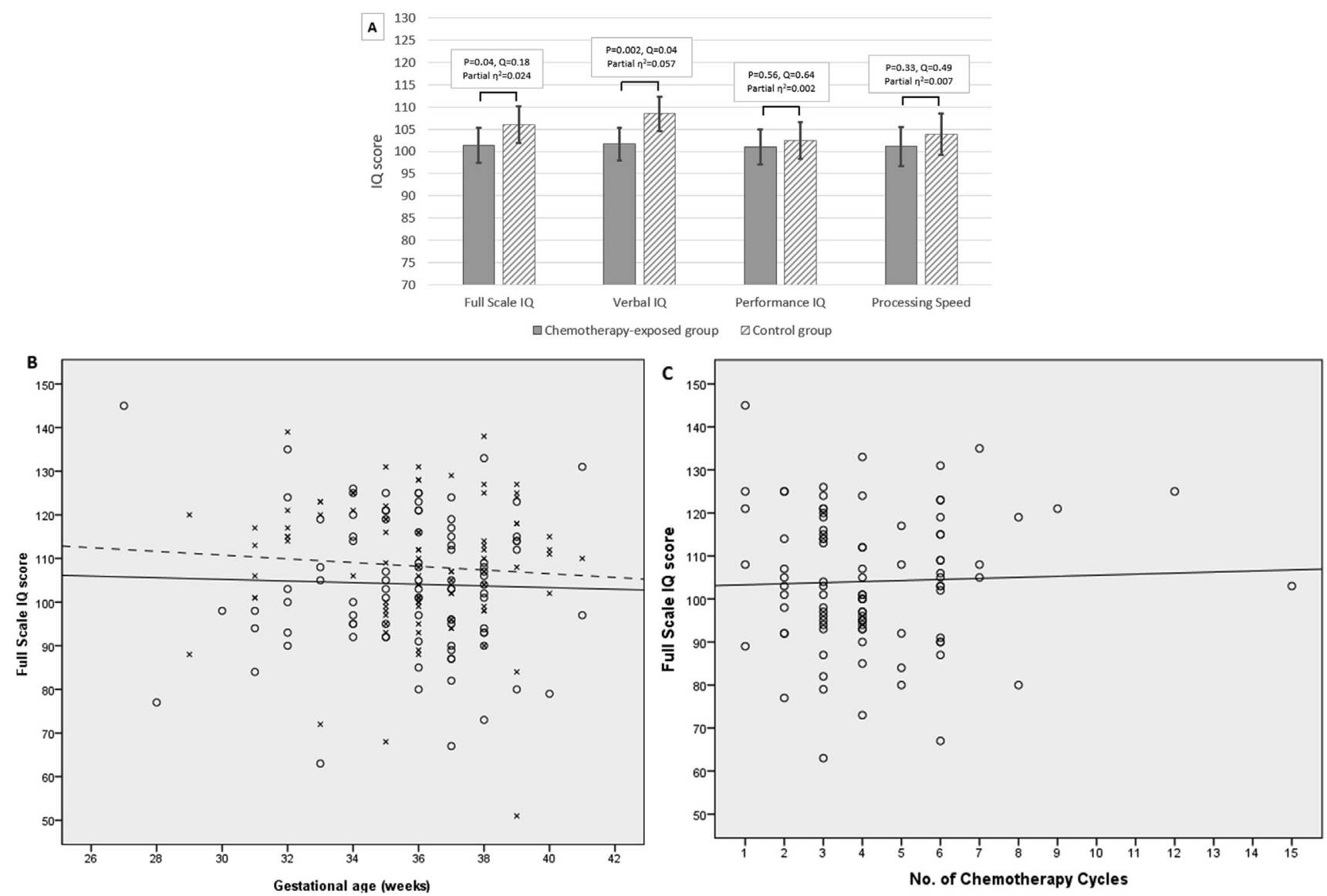

Fig. 2. Cognitive outcome. (A) Comparison of the mean full scale IQ, verbal IQ, performance IQ and processing speed between the chemotherapy-exposed group and the control group. The mean of standardised IQ-scores is 100 with a standard deviation of 15 and scores between 90 and 110 are considered average. Higher scores indicate more advanced development. (B) The relation between full scale IQ and gestational age at birth (in weeks) for the chemotherapy-exposed and control group. Values of children from the chemotherapy-exposed group are represented by circles, and those of children from the control group are represented by crosses. Mean values (as calculated by linear regression) are indicated by a solid line for the chemotherapy-exposed group and a dashed line for the control group. (C) The relation between full scale IQ and the number of chemotherapy cycles administered during pregnancy. (D) Comparison of the raw memory scores from the subtests of the Children's Memory Scale between the chemotherapy-exposed group and the control group. Verbal memory was measured using the subtest numbers (range of scores between 0 and 14 for numbers forward [verbal memory span] and $0-12$ for numbers backward [verbal working memory]). Visuospatial short- and long-term memory was measured using the subtest dot locations (range: 0-6). Visuospatial memory span was measured using the subtest picture locations (range: 0-30). Higher scores indicate more advanced memory skills. (E) Comparison of the standardised T-scores for internalising and externalising behaviour problems on the Child Behavior Checklist between the chemotherapy-exposed group and the control group. The mean of standardised T-scores is 50 with a standard deviation of 15. Higher scores indicate more behaviour problems. (A), (D), (E): The figures show estimated marginal means with standard errors of the means for each group and variable. Raw P values and false discovery rate adjusted $\mathrm{P}$ values (Q values) are presented.

between-group differences in performance IQ or processing speed (Fig. 2A) (eTables 13-14 in the data article). With regard to memory, the difference in estimated marginal means of visuospatial long-term memory was statistically significant, with lower values in the study group (3.9, 95\% CI 3.6 to 4.3$)$ than the control group (4.5, 95\% CI: 4.1 to 4.9$)(\mathrm{P}=0.005, \mathrm{Q}=0.045)$ and in chemotherapy-exposed children (4.0, 95\% CI: 3.3 to 4.8$)$ than their controls (4.7, 95\% CI: 3.9 to 5.5$)$ $(\mathrm{P}=0.005, \mathrm{Q}=0.045)$ (eTables $17-18$ in the data article). No statistically significant differences were found in memory span, short-term memory, attention or behaviour problems between the study and control group and between chemotherapy-exposed and control children (Fig. 2D and E) (eTable 17-22 in the data article). The differences in internalising and externalising behaviour problems were also not statistically significant for study children whose mothers died and those with surviving mothers compared with their matched controls (eTable 23 in the data article).

\subsection{Cardiac evaluation}

Cardiac evaluation was performed in 78 chemotherapyexposed children and matched controls. Median age was 6.1 and 6.2 years in the chemotherapy-exposed and 


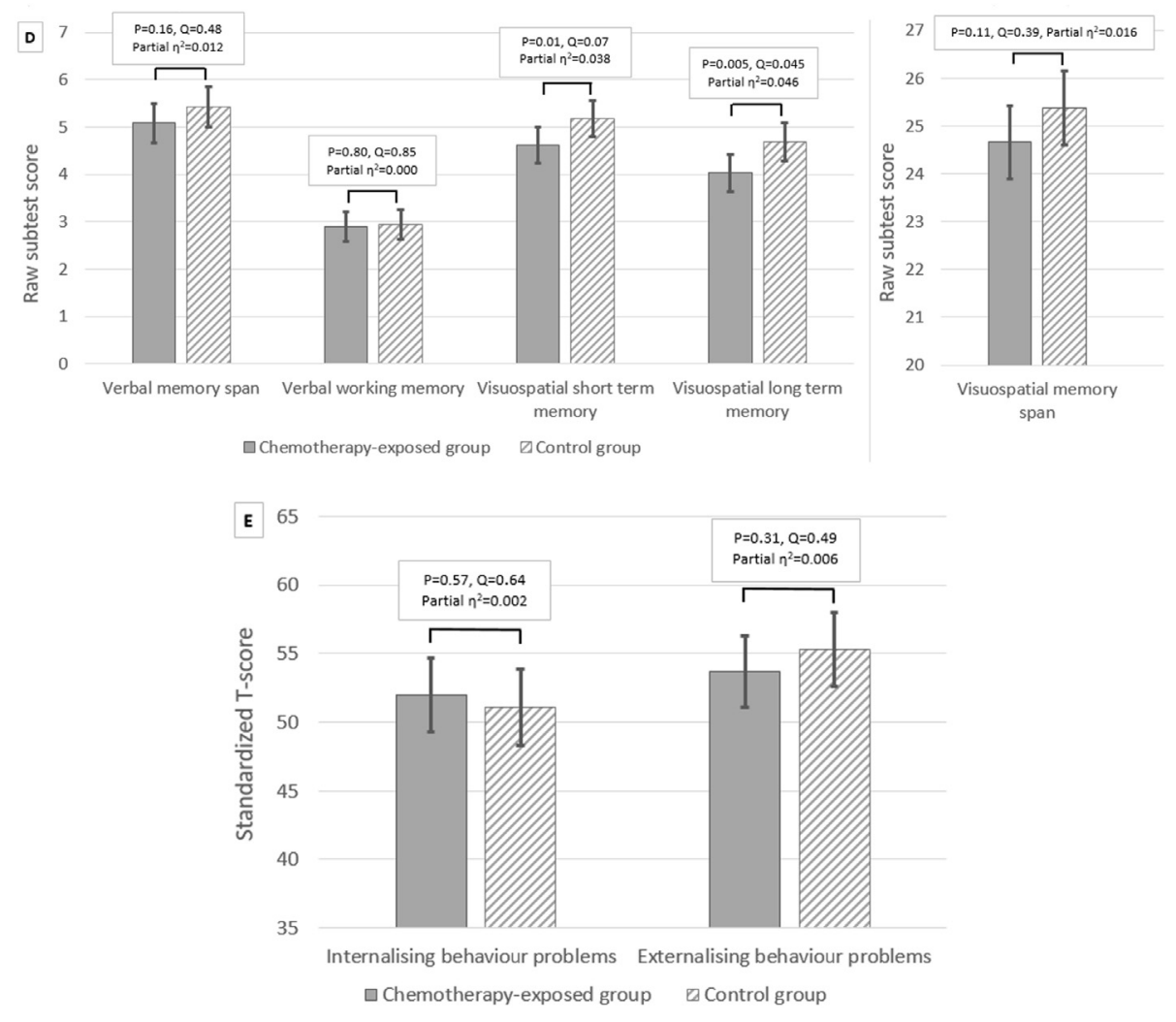

Fig. 2. (continued).

control group, respectively. No statistically significant between-group differences in body surface area, heart rate and systolic blood pressure were found. The difference in means of diastolic blood pressure was statistically significant, with higher values in chemotherapyexposed $(61.1,95 \%$ CI: 59.0 to 63.2$)$ versus control children $(56.0,95 \% \mathrm{CI}: 54.1$ to 57.8$)(\mathrm{P}<0.001$, $\mathrm{Q}<0.001)$ and in a subgroup of 59 anthracyclineexposed (61.8, 95\% CI: 59.3 to 64.4) versus control children (55.9, 95\% CI: 53.6 to 58.1) (P $<0.001$, $\mathrm{Q}=0.02$ ) (Table 4 and eTable 24 in the data article). Electrocardiographic evaluation did not reveal rhythm or conduction abnormalities. On echocardiographic examination, no structural abnormalities were detected in any of the children. The difference in means of the primary outcome left ventricular shortening fraction was not statistically significant between chemotherapyexposed and control children. In addition, no statistically significant between-group differences were found in secondary outcomes.

\subsection{Health problems}

The incidence of health problems and the need for surgery or care as reported by the parents were mostly comparable between the study and control group, but children from the study group were 3 times more likely to wear glasses than the controls (14.9 versus 5.0\%)
(eTable 25 in the data article). Of 14 children exposed to cisplatin, hearing loss was determined in 3 of 8 children with available audiometric data (eTable 26 in the data article). General paediatric and clinical neurological examinations were normal in 96 of 103 study children (93.2\%) undergoing examination (eTable 27 in the data article).

\section{Discussion}

In this multicenter prospective cohort study, cognitive development, health problems and growth were compared between 132 children born to mothers diagnosed with cancer during pregnancy and non-exposed matched controls and between a subgroup of 97 chemotherapy-exposed children and controls. The cardiac structure and function were also evaluated in 78 chemotherapy-exposed children and controls.

The differences in cognitive outcomes on most tests were not statistically significant between the study and control group and between the chemotherapy-exposed subgroup and controls. Especially, there were no statistically significant between-group differences in the primary outcome full scale IQ. In addition, full scale IQ was not related to the number of chemotherapy cycles administered during pregnancy or to the estimated foetal dose of radiation. No statistically significant differences were found in performance IQ and processing 


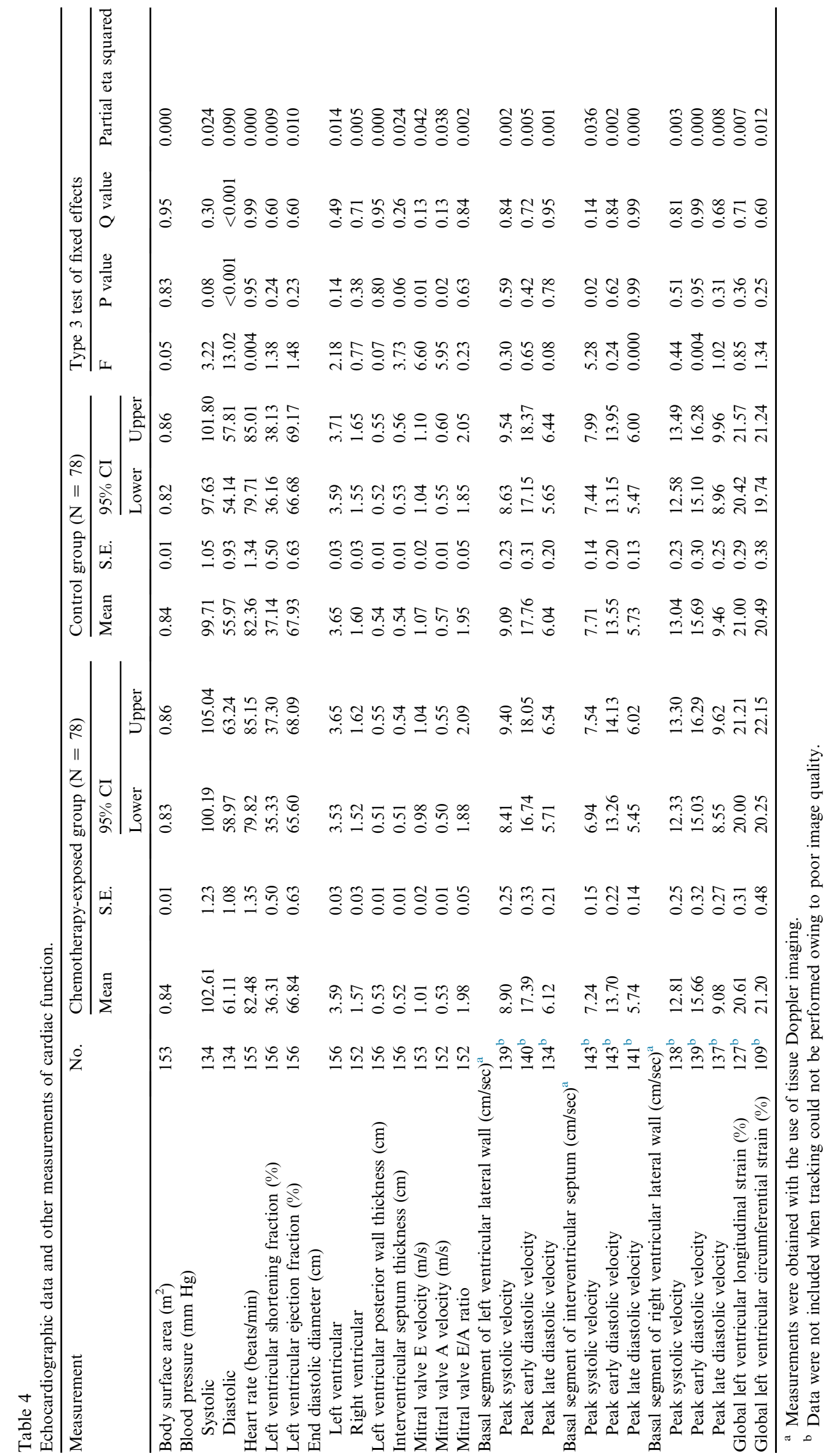


speed. However, children from the study group and children exposed to chemotherapy scored on average 6 points lower on verbal IQ than their matched controls. Although the difference was statistically significant, the clinical relevance may be limited as the values were within the normal range and the between-group difference was smaller than one standard deviation (i.e. 15 IQ points). A study in preterm infants showed that increased amount of adult talk during the neonatal intensive care unit stay may contribute to higher cognitive and language outcomes at 7 and 18 months corrected age [11]. In the case of cancer during pregnancy, mother-child interactions in the neonatal period and early years of life may be more restricted because of the maternal disease and treatment or even absent in the case of maternal death. Our data support this hypothesis, as verbal IQ was more affected in children whose mothers died than in children with surviving mothers. Furthermore, the visuospatial long-term memory score was significantly lower in the study group and in the chemotherapy-exposed subgroup than those in their matched controls, although attention, memory span and short-term memory were not affected. This is in contrast with studies on childhood cancer survivors mostly reporting working memory and attention deficits and slower information processing speed [12].

Notwithstanding the encountered differences in verbal IQ and visuospatial long-term memory and given the large range of cognitive functions assessed in this study, most cognitive functions were normal at the age of 6 years. This is largely consistent with our previous findings in the 1.5-3 years cohort and other studies, reporting minor to no statistically significant differences or results within normal ranges [5,6,13-15].

In our study, $60.6 \%$ of children were born preterm, which may result from elective induction of delivery as part of treatment strategies to limit ongoing exposure of the foetus to cancer treatment or from spontaneous preterm labour which may have various cancer-related and cancer-non-related causes. In the 1.5-3 years cohort, prematurity was associated with a worse cognitive outcome [6]. This relationship was no longer present at the age of 6 years with regard to full scale IQ. Inconsistent findings have been reported on the longterm effects of preterm birth on cognition, especially for late preterm born children, who are the most represented preterm born children in our study [16-19].

The cardiac evaluation in chemotherapy-exposed children was overall reassuring. No statistically significant between-group differences in cardiac dimension and global function measurements including tissue Doppler imaging and strain analyses were found, and all measurements were within normal ranges. However, the diastolic blood pressure was higher in chemotherapyexposed and anthracycline-exposed versus control children, but the clinical relevance may be limited. The overall normal cardiac findings are consistent with our previous findings in the 3 -year-old cohort and other studies [6,20-22].

The incidence of health problems was comparable between study and control children, but children from the study group were three times more likely to wear glasses than the controls. An association with cancer treatment is possible as the development of the eyes and central nervous system takes place throughout the entire pregnancy and needs further investigation. In addition, three children exposed to cisplatin were diagnosed with hearing loss. Cisplatin has also been related to ototoxicity in adults and children with cancer [23]. Ototoxicity is associated with declines on intellectual and academic performances and worse social and langauge development [24,25]. Where possible, cisplatin should be replaced by carboplatin with a more favourable toxicity profile. Long-term surveillance of auditory function of children prenatally exposed to platinum-based treatment is recommended.

Our study has some limitations. As cancer may have been present before the start of pregnancy and/or in some cases termination of pregnancy is indicated or preferred by the couple, a selection bias may be present towards a healthier subset of the eligible population and malignancies not necessitating chemotherapy during the first trimester. The results cannot be extrapolated to all types of chemotherapeutic agents and to all trimesters of pregnancy. Individual drug evaluation was not possible because of the frequent combination of different cancer treatments.

\section{Conclusions}

Children prenatally exposed to maternal cancer, the associated stress, diagnostic imaging and treatments have cognitive and cardiac outcomes within normal ranges at the age of 6 years. However, they are at risk for lower verbal IQ and visuospatial long-term memory scores and for higher diastolic blood pressure than matched controls. In addition, they are at higher risk for need for glasses and ototoxicity in case of cisplatin exposure. In accordance with earlier studies, our data show that in many cases, the risks of maternal cancer treatment during pregnancy do not outweigh the benefit of maternal treatment delay or the need for termination of pregnancy. The results of our study will help patients to make well-informed decisions.

\section{Role of the funding source}

This research was supported by European Union's Horizon 2020 research and innovation programme (No. 647047), Research Foundation-Flanders, Stichting tegen Kanker, Belgian Cancer Plan, Koningin Wilhelmina Fonds, Kom Op Tegen Kanker, Stichting Mitialto and 
Charles University grant PROGRES Q-34. The funding sources had no involvement in the study design, in the collection, analysis and interpretation of data, in the writing of the report and in the decision to submit the article for publication.

\section{Conflict of interest statement}

None declared.

\section{Acknowledgements}

The authors are grateful to Jeroen Blommaert, Jana Dekrem, Frederic Goffin, Vincent Rigo, Camilla Fontana, Fabio Mosca, Sofia Passera, Odoardo Picciolini, Giovanna Scarfone, Fedro Alessandro Peccatori, Maria Lucia Boffi, Martina Delle Marchette, Renata Nacinovich, Christianne Lok, Vera Wolters, Ingrid Boere, Els Witteveen, Carolina Schröder, Christianne de Groot, Martine van Grotel, Marry van den Heuvel-Eibrink, Anna Babkova and Vít Drochýtek, all members of the International Network on Cancer, Infertiliy and Pregnancy (INCIP). The authors are grateful to Ilse De Croock, Griet De Mulder, Ilse Denolf, Tom Depuydt, Liesbeth Leemans, Kaat Philippe, Marlies Potoms, Cettina Schellens, Jelle Stans, Caroline Sterken, Lore Vallaeys, Marie-Astrid Van Hoorick, Diane Wolput, Heidi Wouters, Griet Van der Perre, Frédéric Studzinsky, Livia Kapusta, Michel Willemsen, Alice van DijkLokkart, Agnetha Fruijtier, Jitka Havelkova and Wei Hui, their collaborating partners. The authors are grateful to the European Society of Gynaecological Oncology (ESGO) for their continued support.

\section{References}

[1] de Haan J, Verheecke M, Van Calsteren K, et al. Oncological management and obstetric and neonatal outcomes for women diagnosed with cancer during pregnancy: a 20-year international cohort study of 1170 patients. Lancet Oncol 2018 Mar;19(3): 337-46.

[2] Van Calsteren K, Verbesselt R, Beijnen J, et al. Transplacental transfer of anthracyclines, vinblastine, and 4-hydroxy-cyclophosphamide in a baboon model. Gynecol Oncol 2010;119:594-600.

[3] Van Calsteren K, Verbesselt R, Devlieger R, et al. Transplacental transfer of paclitaxel, docetaxel, carboplatin, and trastuzumab in a baboon model. Int J Gynecol Canc 2010;20:1456-64.

[4] Vandenbroucke T, Verheecke M, Fumagalli M, Lok C, Amant F. Effects of cancer treatment during pregnancy on fetal and child development. Lancet Child Adolesc Health 2017;1:302-10.

[5] Amant F, Van Calsteren K, Halaska MJ, et al. Long-term cognitive and cardiac outcomes after prenatal exposure to chemotherapy in children aged 18 months or older: an observational study. Lancet Oncol 2012;13:256-64.

[6] Amant F, Vandenbroucke T, Verheecke M, et al. Pediatric outcome after maternal cancer diagnosed during pregnancy. $\mathrm{N}$ Engl J Med 2015;373:1824-34.
[7] Patane S. Cardiotoxicity: anthracyclines and long term cancer survivors. Int J Cardiol 2014;176:1326-8.

[8] Franco VI, Lipshultz SE. Cardiac complications in childhood cancer survivors treated with anthracyclines. Cardiol Young 2015; 25(Suppl 2):107-16.

[9] Glickman ME, Rao SR, Schultz MR. False discovery rate control is a recommended alternative to Bonferroni-type adjustments in health studies. J Clin Epidemiol 2014;67:850-7.

[10] Blencowe H, Cousens S, Oestergaard MZ, et al. National, regional, and worldwide estimates of preterm birth rates in the year 2010 with time trends since 1990 for selected countries: a systematic analysis and implications. Lancet 2012;379:2162-72.

[11] Caskey M, Stephens B, Tucker R, Vohr B. Adult talk in the NICU with preterm infants and developmental outcomes. Pediatrics 2014;133:e578-84.

[12] Campbell LK, Scaduto M, Sharp W, et al. A meta-analysis of the neurocognitive sequelae of treatment for childhood acute lymphocytic leukemia. Pediatr Blood Canc 2007;49:65-73.

[13] Cardonick EH, Gringlas MB, Hunter K, Greenspan J. Development of children born to mothers with cancer during pregnancy: comparing in utero chemotherapy-exposed children with nonexposed controls. Am J Obstet Gynecol 2015;212.

[14] Aviles A, Neri N. Hematological malignancies and pregnancy: a final report of 84 children who received chemotherapy in utero. Clin Lymphoma 2001;2:173-7.

[15] Hahn KM, Johnson PH, Gordon N, et al. Treatment of pregnant breast cancer patients and outcomes of children exposed to chemotherapy in utero. Cancer 2006;107:1219-26.

[16] Odd DE, Emond A, Whitelaw A. Long-term cognitive outcomes of infants born moderately and late preterm. Dev Med Child Neurol 2012;54:704-9.

[17] Bhutta AT, Cleves MA, Casey PH, Cradock MM, Anand KJ. Cognitive and behavioral outcomes of school-aged children who were born preterm: a meta-analysis. J Am Med Assoc 2002;288: $728-37$.

[18] de Jong M, Verhoeven M, van Baar AL. School outcome, cognitive functioning, and behaviour problems in moderate and late preterm children and adults: a review. Semin Fetal Neonatal Med 2012;17:163-9.

[19] Talge NM, Holzman C, Wang J, Lucia V, Gardiner J, Breslau N. Late-preterm birth and its association with cognitive and socioemotional outcomes at 6 years of age. Pediatrics 2010;126: 1124-31.

[20] Aviles A, Neri N, Nambo MJ. Long-term evaluation of cardiac function in children who received anthracyclines during pregnancy. Ann Oncol 2006;17:286-8.

[21] Aviles A, Nambo MJ, Huerta-Guzman J, Neri N, Cleto S. Speckle-tracking echocardiography to detect cardiac toxicity in children who received anthracyclines during pregnancy. Clin Lymphoma, Myeloma \& Leukemia 2016;16:1-4.

[22] Gziri MM, Hui W, Amant F, et al. Myocardial function in children after fetal chemotherapy exposure. A tissue Doppler and myocardial deformation imaging study. Eur J Pediatr 2013;172: $163-70$.

[23] Yancey A, Harris MS, Egbelakin A, Gilbert J, Pisoni DB, Renbarger J. Risk factors for cisplatin-associated ototoxicity in pediatric oncology patients. Pediatr Blood Canc 2012;59:144-8.

[24] Clemens E, de Vries AC, Pluijm SF, et al. Determinants of ototoxicity in 451 platinum-treated Dutch survivors of childhood cancer: a DCOG late-effects study. Eur J Canc 2016;69:77-85.

[25] Olivier TW, Bass JK, Ashford JM, et al. Cognitive implications of ototoxicity in pediatric patients with embryonal brain tumors. J Clin Oncol 2019;37:1566-75.

[26] Wechsler D. Wechsler preschool and primary Scale of intelligence. 3rd ed. San Antonio, TX: Psychological Corporation; 2002. 
[27] Cohen MJ. Children's memory Scale. Paris: Les Editions du Centre de Psychologie Appliquée; 1997.

[28] de Sonneville LMJ. Amsterdam neuropsychological tasks: a computer-aided assessment program. In: den Brinker BPLM, Beek PJ, Brand AN, Maarse SJ, Mulder LJM, editors. Cogntive ergonomics, clinical assessment and computer-assisted learning: computers in psychology. Lisse: Swets \& Zeitlinger; 1999. p. 187-203.

[29] Achenbach TM, Rescorla LA. Manual for the ASEBA schoolage forms \& profiles. Burlington, VT: University of Vermont, Research Center for Children, Youth, \& Families; 2001. 